\title{
COMPUTATIONAL FLUID DYNAMICS / MONTE CARLO SIMULATION OF DUSTY GAS FLOW IN A "ROTOR-STATOR" SET OF AIRFOIL CASCADES
}

\section{Yu. M. Tsirkunov and D. A. Romanyuk}

\author{
Baltic State Technical University \\ Department of Plasma- and Gas Dynamics and Heat Engineering \\ 1 Krasnoarmeiskaya 1st Str., St. Petersburg 190005, Russia
}

\begin{abstract}
A dusty gas flow through two, moving and immovable, cascades of airfoils (blades) is studied numerically. In the mathematical model of two-phase gas-particle flow, the carrier gas is treated as a continuum and it is described by the Navier-Stokes equations (pseudo-DNS (direct numerical simulation) approach) or the Reynolds averaged Navier-Stokes (RANS) equations (unsteady RANS approach) with the Menter $k-\omega$ shear stress transport (SST) turbulence model. The governing equations in both cases are solved by computational fluid dynamics (CFD) methods. The dispersed phase is treated as a discrete set of solid particles, the behavior of which is described by the generalized kinetic Boltzmann equation. The effects of gas-particle interaction, interparticle collisions, and particle scattering in particle-blade collisions are taken into account. The direct simulation Monte Carlo (DSMC) method is used for computational simulation of the dispersed phase flow. The effects of interparticle collisions and particle scattering are discussed.
\end{abstract}

\section{INTRODUCTION}

Military aircraft can sometimes operate in dusty atmosphere. Civilian airplanes can fly into a cloud of volcanic ash. In all these cases, one deals with a gasparticle flow inside engine ducts. Dispersed particles suspended in a high-speed inlet flow, being more inertial than the carrier gas, can collide with blades of a fan, a compressor, and even a turbine of a turbofan (or turbojet) engine resulting in abrasive erosion and quick failure of blades. The erosive effect from particles can reduce the lifetime of blades and, hence, of the whole engine, up to a factor 
of 10. For this reason, the problem of dusty gas flow inside aircraft engines has been the focus of attention for at least 40 previous years.

Blades of a fan and an inlet stage of a low-pressure compressor are extremely subjected to damage by high-speed particles' impacts. Modeling and simulation of dusty gas flow through rotating rows of blades is a very complex problem. Strictly speaking, a time-dependent flow is three-dimensional (3D). However, many key flow features in the sequential rows of blades can be studied using a two-dimensional (2D) flow model in the plane, which represents a developed mean-circle cross section. Such an approach turned out to be very fruitful for flow analysis in axial-flow compressors and turbines and it is well known as the 2D theory of cascade flow (see, e.g., [1]). Stationary 2D flow of dilute gasparticle mixture with regular motion of monosized particles through a turbine cascade was studied numerically in [2] with special emphasis on application of the full Lagrangian method, which gives very high resolution of the particle concentration field even in the case when singular surfaces with infinite concentration appear in the flow. Such singularities are typical for the flow model taken in [2]. However, these singularities disappear if some random in nature effects such as interparticle collisions, particle scattering in particle-blade collisions, and particle size distribution are taken into account. Unsteady dusty gas flow through a "rotor-stator" cascade with taking account of particle size distribution and particle scattering was studied in [3].

It is quite evident that if the particle concentration in the flow is vanishingly low, the reverse effect of particles on the carrier gas is negligible and collisions between particles play no role. Estimations obtained in [4] for a flow with coarse-grained dispersed particles over a blunt body show that the collisions between incident and rebounded particles begin to play a noticeable role at a much lower particle concentration in an undisturbed flow (at particle volume fraction $\alpha_{p \infty} \sim 10^{-5}$ ) than the concentration at which the reverse effect becomes important. Closer examination showed that the effect of particles on a carrier gas flow is determined not only by the particle concentration, but also by the relative particle inertia, which is characterized by the Stokes number. In the present study, the authors assume that $\alpha_{p \infty}$ is low enough to neglect the reverse effect, but the interparticle collisions are taken into account. The main objective is to estimate the role of collisions in the redistribution of the dispersed phase in the flow.

A kinetic model for description of a collisional "gas" of particles was proposed in [5]. In this model, the dispersed phase is treated as a set of a large number of particles and its state is described by the one-particle distribution function, similarly to the theory of rarefied gas dynamics. The generalized Bolzmann equation derived in [5] takes account of the carrier gas action on particles and frictional and noncompletely elastic collisions between particles. To computationally simulate the behavior of the collisional "gas" of particles, the DSMC algorithm was developed in [6]. In the present work, it was adapted for numerical study of the particle phase flow through airfoil cascades. 


\section{ARRANGEMENT OF CASCADES AND SCHEMATIC OF THE FLOW}

Consider a two-phase gas-particle flow through a set of two cascades (Fig. 1). The first cascade moves at constant velocity $V_{r}$ in the direction normal to the undisturbed flow and the second cascade is fixed. Both cascades have the same pitch $s$ (the distance between airfoils along the cascade). The airfoils of the first cascade are set at angle $\beta$. For visualizing the particle-phase flow in the computational simulation, a cloud of particles of finite width $h$ was considered.

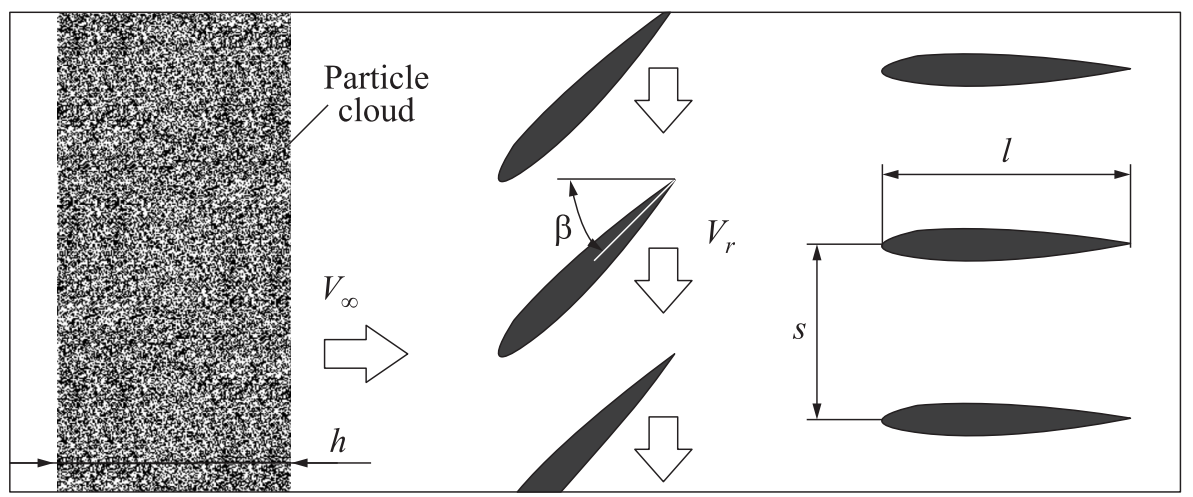

Figure 1 Arrangement of cascades in an undisturbed flow

Consider the two-phase flow as one-way coupled. This means that the effect of the dispersed phase on the carrier gas can be ignored. In this case, modeling of gas-particle flow can be reduced to two sequential steps: modeling of the carrier gas flow without taking the dispersed phase into account and calculation of the particle's motion in the predetermined gas flow field. Although the gas flow is $2 \mathrm{D}$, the motion of each particle is considered as $3 \mathrm{D}$ because $3 \mathrm{D}$ effects are important for the interparticle collisions and scattering of particles in particleblade collisions.

\section{MODELING OF THE CARRIER GAS FLOW}

Comparison of numerical results obtained for cascade flow on the basis of inviscid and viscous gas flow models clearly demonstrated that the viscous effects play an important role [3]. The flow through cascades is usually turbulent. In the present study of the flow through a "rotor-stator" set of cascades, two flow models were used to simulate the flow field: $(i)$ one based on complete Navier-Stokes 
equations (that corresponds to the pseudo-DNS approach, because the flow was considered as 2D, and the grid was too coarse for high resolution of turbulent flow structure), and (ii) another based on the unsteady RANS equations (URANS approach) with the Menter $k-\omega$ SST turbulence model $[7,8]$.

The Navier-Stokes equations for time-dependent compressible 2D flow can be written in Cartesian coordinates $(x, y)$ in the following compact form [9]:

$$
\frac{\partial \mathbf{Q}}{\partial t}+\frac{\partial \mathbf{F}_{x}}{\partial x}+\frac{\partial \mathbf{F}_{y}}{\partial y}=\frac{\partial \mathbf{G}_{x}}{\partial x}+\frac{\partial \mathbf{G}_{y}}{\partial y}
$$

where vectors $\mathbf{Q}, \mathbf{F}_{x}, \mathbf{F}_{y}, \mathbf{G}_{x}$, and $\mathbf{G}_{y}$ are defined as follows:

$$
\begin{gathered}
\mathbf{Q}=\left(\begin{array}{c}
\rho \\
\rho u \\
\rho v \\
\rho e
\end{array}\right) ; \quad \mathbf{F}_{x}=\left(\begin{array}{c}
\rho u \\
\rho u^{2}+p \\
\rho u v \\
(\rho e+p) u
\end{array}\right) ; \quad \mathbf{F}_{y}=\left(\begin{array}{c}
\rho v \\
\rho u v \\
\rho v^{2}+p \\
(\rho e+p) v
\end{array}\right) ; \\
\mathbf{G}_{x}=\left(\begin{array}{c}
0 \\
0 \\
\tau_{x x} \\
\tau_{x y} \\
\tau_{y y} \\
u \tau_{x x}+v \tau_{x y}-q_{x}
\end{array}\right) ; \quad \mathbf{G}_{y}=\left(\begin{array}{c} 
\\
u \tau_{x y}+v \tau_{y y}-q_{y}
\end{array}\right) .
\end{gathered}
$$

Here,

$$
\begin{gathered}
\tau_{x x}=\frac{2}{3} \mu\left(2 \frac{\partial u}{\partial x}-\frac{\partial v}{\partial y}\right) ; \quad \tau_{y y}=\frac{2}{3} \mu\left(2 \frac{\partial v}{\partial y}-\frac{\partial u}{\partial x}\right) ; \tau_{x y}=\mu\left(\frac{\partial u}{\partial y}+\frac{\partial v}{\partial x}\right) \\
q_{x}=-\lambda \frac{\partial T}{\partial x} ; \quad q_{y}=-\lambda \frac{\partial T}{\partial y} ; \quad p=\rho R T ; \quad e=c_{v} T+\frac{u^{2}+v^{2}}{2}
\end{gathered}
$$

In these equations, $t$ is the time; $u$ and $v$ are the $x$ - and $y$-components of the velocity vector; $\rho, p, e, T, \mu$, and $\lambda$ are the gas density, pressure, specific total energy, temperature, viscosity, and thermal conductivity, respectively; $R$ is the gas constant; and $c_{v}$ is the specific heat at constant volume. For $\mu$ and $\lambda$, the following relations were used: $\mu=\mu_{s}\left(T / T_{s}\right)^{3 / 2}\left(T_{s}+C_{s}\right) /\left(T+C_{s}\right)$ and $\lambda$ $=c_{p} \mu / \operatorname{Pr}$ where the first relation is the Sutherlend formula (for air, $\mu_{s}=1.71$. $10^{-5} \mathrm{~N} \cdot \mathrm{s} / \mathrm{m}^{2}, T_{s}=288 \mathrm{~K}$, and $\left.C_{s}=117 \mathrm{~K}\right) ; \operatorname{Pr}$ is the Prandtl number; and $c_{p}$ is the specific heat at constant pressure. The system of Eqs. (1)-(4) is closed.

The full system of equations for the URANS approach with the Menter $k-\omega$ SST turbulence model is very cumbersome $[7,8]$ and is not given here.

\section{MODELING OF THE PARTICLE-PHASE MOTION}

The motion of particles is governed by the gas-particle interaction, interparticle collisions, and particle-blade collisions. The particles are more inertial than the 
carrier gas and, therefore, they do not follow the streamlines and can collide with blades and rebound (reflect) from them. The reflected particles can collide with the incident ones; these collisions, being random in nature, result in chaotic motion of particles. In the classical two-phase flow theory, particles are assumed to have a spherical shape; however, real particles (sand, ash, etc.) are not spherical and because of this, they are scattered in particle-wall collisions. The particle scattering in cascade flows was analyzed in [3] and it is taken into account in the present study.

\subsection{Kinetic Model of the Collisional "Gas" of Particles}

Here, the dispersed phase is considered as a discrete set of solid particles which move in the carrier gas and collide with each other. Below, the kinetic model for the collisional "gas" of dispersed particles proposed in [5] is briefly described. It is assumed that particles are spheres of the same radius $r_{p}$. They interact with each other only through binary collisions that is valid if the "gas" of particles is not too dense. Besides, it is assumed that collision process is instantaneous and the position of colliding particles does not change during the collision. The state of the $i$ th particle is determined by a point $\mathbf{x}_{i}$ in the phase space which includes the particle position vector $\mathbf{r}_{i}$, the particle translational and rotational velocities $\left(\mathbf{v}_{p i}\right.$ and $\boldsymbol{\omega}_{p i}$, respectively), i. e., $\mathbf{x}_{i}=\left(\mathbf{r}_{i}, \mathbf{v}_{p i}, \boldsymbol{\omega}_{p i}\right)$. The vector $\mathbf{x}_{i}$ is split into $\mathbf{r}_{i}$ and $\mathbf{y}_{i}=\left(\mathbf{v}_{p i}, \boldsymbol{\omega}_{p i}\right)$. Denote the parameters of the $i$ th and $j$ th particles before and after their collision by the superscripts "minus" and "plus," respectively. The postcollisional parameters of colliding particles are fully determined by the precollisional ones and the relative position of particles at the collision instant; thus, one can write $\mathbf{y}_{k}^{+}=\mathbf{y}_{k}^{+}\left(\mathbf{y}_{i}^{-}, \mathbf{y}_{j}^{-}, \mathbf{n}_{i j}\right), \quad k=i, j$, where $\mathbf{n}_{i j}$ is the unit vector directed from the center of the $i$ th particle towards the center of the $j$ th particle at the collision instant. The above relation for $\mathbf{y}_{k}^{+}$is presumed to give a one-to-one correspondence between $\mathbf{y}_{k}^{-}$and $\mathbf{y}_{k}^{+}$, so that the Jacobian $J_{1}=\left|D\left(\mathbf{y}_{i}^{+}, \mathbf{y}_{j}^{+}\right) / D\left(\mathbf{y}_{i}^{-}, \mathbf{y}_{j}^{-}\right)\right| \neq 0$ and, hence, the relation can be resolved for the particle parameters before the collision $\mathbf{y}_{k}^{-}=\mathbf{y}_{k}^{-}\left(\mathbf{y}_{i}^{+}, \mathbf{y}_{j}^{+}, \mathbf{n}_{i j}\right), \quad k=i, j$. A collision is physically feasible only when $\mathbf{g}_{i j}^{-} \cdot \mathbf{n}_{i j} \leq 0$ where $\mathbf{g}_{i j}=\mathbf{v}_{j}-\mathbf{v}_{i}$. Also, assume that states of any two particles in the phase space are not statistically correlated. This assumption is valid if the mean free path of particles moving in the carrier gas is much smaller than the particle momentum response length. These assumptions and reasoning are similar to those accepted in rarefied gas dynamics for collisions between molecules.

Let $f_{1}=f\left(\mathbf{x}_{1}, t\right)$ be the distribution function such that

$$
f_{1} d \mathbf{x}_{1}=f\left(\mathbf{r}_{1}, \mathbf{v}_{p 1}, \boldsymbol{\omega}_{p 1}, t\right) d \mathbf{r}_{1} d \mathbf{v}_{p 1} d \boldsymbol{\omega}_{p 1}
$$

is the number of particles with coordinates and velocities from the elementary volume $d \mathbf{r}_{1} d \mathbf{v}_{p 1} d \boldsymbol{\omega}_{p 1}$ in the vicinity of point $\mathbf{x}_{1}=\left(\mathbf{r}_{1}, \mathbf{v}_{p 1}, \boldsymbol{\omega}_{p 1}\right)$ in the phase 
space. Then, the following kinetic Boltzmann-type equation for $f_{1}$ can be derived:

$$
\frac{\partial f_{1}}{\partial t}+\frac{\partial}{\partial \mathbf{r}_{1}}\left(\mathbf{v}_{p 1} f_{1}\right)+\frac{\partial}{\partial \mathbf{v}_{p 1}}\left(\frac{\mathbf{f}_{p 1}}{m_{p}} f_{1}\right)+\frac{\partial}{\partial \boldsymbol{\omega}_{p 1}}\left(\frac{\mathbf{I}_{p 1}}{I_{p}} f_{1}\right)=I_{\text {coll }} .
$$

This equation is a particular case of the more general kinetic equation [5] which also takes gas-particle heat transfer and particle size distribution into account. The collisional integral in the right-hand side is given by

$$
I_{\text {coll }}=4 r_{p}^{2} \int d \mathbf{y}_{2} \int_{\mathbf{g}_{12} \cdot \mathbf{n}_{12} \leq 0}\left(\frac{f_{1}^{-} f_{2}^{-}}{J}-f_{1} f_{2}\right)\left|\mathbf{g}_{12} \cdot \mathbf{n}_{12}\right| \sin \chi_{12} d \chi_{12} d \varepsilon_{12}
$$

where

$$
J=\left|\frac{\mathbf{g}_{12} \cdot \mathbf{n}_{12}}{\mathbf{g}_{12}^{-} \cdot \mathbf{n}_{12}} J_{1}\right| .
$$

Here, $m_{p}$ and $I_{p}$ are the particle mass and moment of inertia; $\mathbf{f}_{p 1}$ and $\mathbf{I}_{p 1}$ are the force and the torque acting on a particle from the carrier gas which are calculated for the particle with the parameters $\left(\mathbf{v}_{p 1}, \boldsymbol{\omega}_{p 1}\right)$ at point $\mathbf{r}_{1}$ in the flow; $f_{2}=f\left(\mathbf{r}_{1}, \mathbf{y}_{2}, t\right) ; f_{1}^{-}=f\left(\mathbf{r}_{1}, \mathbf{y}_{1}^{-}, t\right) ; f_{2}^{-}=f\left(\mathbf{r}_{1}, \mathbf{y}_{2}^{-}, t\right) ; \mathbf{g}_{12}=\mathbf{v}_{p 2}-\mathbf{v}_{p 1} ;$ and $\mathbf{n}_{12}$ has been defined above. The inequality $\mathbf{g}_{12} \cdot \mathbf{n}_{12} \leq 0$ is the condition of physical feasibility of a collision between the first and the second particles, and the angles $\chi_{12}$ and $\varepsilon_{12}$ specify the direction of $\mathbf{n}_{12}$ in spherical coordinates with the origin at the center of the first particle [5].

The function $J$ which enters into the collisional integral is given by Eq. (7). It depends on the particle-particle collision model which will be discussed later.

Let $\Phi=\Phi\left(\mathbf{x}_{i}\right)$ be a parameter of an individual particle. If a hydrodynamic parameter of a "gas" of particles (in other words, macroparameter of the dispersed phase) $\langle\Phi\rangle(\mathbf{r}, t)$ at point $\mathbf{r}$ in the physical space is defined as the ensemble averaged value of $\Phi=\Phi\left(\mathbf{x}_{i}\right)$ in a unit volume of the gas-particle mixture, then $\langle\Phi\rangle(\mathbf{r}, t)$ can be expressed in terms of $\Phi$ and $f\left(\mathbf{x}_{1}, t\right)$ as follows:

$$
\langle\Phi\rangle(\mathbf{r}, t)=\int \Phi\left(\mathbf{r}, \mathbf{y}_{1}, t\right) f\left(\mathbf{r}, \mathbf{y}_{1}, t\right) d \mathbf{y}_{1} .
$$

For example, the particle number density $n_{p}$, the particle volume fraction $\alpha_{p}$, the hydrodynamic velocity $\mathbf{w}_{p}$, and the specific energy of particle chaotic motion $e_{p}$ are calculated as follows:

$$
n_{p}=\langle 1\rangle ; \quad \alpha_{p}=\frac{4}{3} \pi r_{p}^{3} n_{p} ; \quad \mathbf{w}_{p}=\frac{\left\langle\mathbf{v}_{p}\right\rangle}{n_{p}} ; \quad e_{p}=\frac{1}{m_{p} n_{p}}\left\langle\frac{m_{p}\left(\mathbf{v}_{p}-\mathbf{w}_{p}\right)^{2}}{2}\right\rangle .
$$

The model of a noncompletely elastic collision between two particles is an important part of the kinetic model of a collisional "gas" of solid particles. Write 
the momentum and angular momentum balance equations for a pair of the $i$ th and the $j$ th colliding spherical particles:

$$
\left.\begin{array}{l}
m_{p} \mathbf{v}_{p i}^{-}+m_{p} \mathbf{v}_{p j}^{-}=m_{p} \mathbf{v}_{p i}^{+}+m_{p} \mathbf{v}_{p j}^{+} \\
I_{p}\left(\boldsymbol{\omega}_{p k}^{+}-\boldsymbol{\omega}_{p k}^{-}\right)=m_{p} r_{p} \mathbf{e}_{k} \times\left(\mathbf{v}_{p k}^{+}-\mathbf{v}_{p k}^{-}\right), \quad k=i, j,
\end{array}\right\}
$$

where $\mathbf{e}_{i}=\mathbf{n}_{i j}, \mathbf{e}_{j}=-\mathbf{n}_{i j}$. The system of three-vector Eqs. (8) is not closed because it involves four unknowns $\mathbf{v}_{p k}^{+}$and $\boldsymbol{\omega}_{p k}^{+}, k=i, j$. Some additional hypotheses for the interaction between particles should be introduced to make this system closed. Considering the relative velocity of particles at the contact point

$$
\mathbf{U}_{i j}=\mathbf{v}_{p j}-\mathbf{v}_{p i}-r_{p}\left(\boldsymbol{\omega}_{p i}+\boldsymbol{\omega}_{p j}\right) \times \mathbf{n}_{i j},
$$

let us represent $\mathbf{U}_{i j}^{+}$in the form

$$
\mathbf{U}_{i j}^{+}=-a_{p n} \mathbf{U}_{i j(n)}^{-}+a_{p t} \mathbf{U}_{i j(t)}^{-}
$$

where $a_{p n}$ and $a_{p t}$ are the restitution coefficients of the normal $\left(\mathbf{U}_{i j(n)}\right.$ $\left.=\left(\mathbf{U}_{i j} \cdot \mathbf{n}_{i j}\right) \mathbf{n}_{i j}\right)$ and tangential $\left(\mathbf{U}_{i j(t)}=\mathbf{U}_{i j}-\mathbf{U}_{i j(n)}\right)$ components of the relative velocity $\mathbf{U}_{i j}$. These coefficients are assumed to take into account the losses of particle kinetic energy due to inelastic deformation during a collision $\left(a_{p n}\right)$ and due to the particle surface roughness $\left(a_{p t}\right)$. Their values lie in the ranges: $0 \leq a_{p n} \leq 1$ and $-1 \leq a_{p t} \leq 1$.

The true values of $a_{p n}$ and $a_{p t}$ in different collision conditions are unknown, and theoretical or experimental determination of them is very difficult. The restitution coefficients have been assumed to be constant. If the values of $a_{p n}$ and $a_{p t}$ are given, then the system of Eqs. (8)-(10) becomes closed and can be solved for the parameters of the $i$ th and $j$ th particles after their collision. In this case, one can also calculate the Jacobian

$$
J_{1}=\left|\frac{D\left(\mathbf{y}_{i}^{+}, \mathbf{y}_{j}^{+}\right)}{D\left(\mathbf{y}_{i}^{-}, \mathbf{y}_{j}^{-}\right)}\right|=-a_{p n} a_{p t}^{2}
$$

and then the parameter $J$ in the collisional integral of Eq. (7): $J=a_{p n}^{2} a_{p t}^{2}$. In reality, the absolute values of $a_{p n}$ and $a_{p t}$ are always less than unity; hence, $J<1$. The multiplier $1 / J$ in the collisional integral takes into account the "compression" of the phase space caused by the losses of the kinetic energy of colliding particles. In calculations, the values of the restitution coefficients were taken as $a_{p n}=0.5$ and $a_{p t}=0.9$.

If particles move in vacuum (i. e., $\mathbf{f}_{p 1}=\mathbf{I}_{p 1}=0$ in Eq. (5)) and collisions between particles are completely elastic (i.e., $a_{p n}=1, a_{p t}=1$, and, hence, $J=1$ ), then one comes to the model of a simple gas of molecules treated as hard spheres. In this case, Eq. (5) transforms to the well-known Boltzmann equation used in the rarefied gas dynamics. 


\subsection{Gas-Particle Interaction}

In the present study, the force $\mathbf{f}_{p}$ acting on a particle included the drag force $\mathbf{f}_{D}$ and the lift Magnus force $\mathbf{f}_{M}\left(\mathbf{f}_{p}=\mathbf{f}_{D}+\mathbf{f}_{M}\right)$ which dominate over all other force components in the flow under consideration. These forces and the torque $\mathbf{I}_{p}$ can be expressed in terms of the dimensionless coefficients $C_{D}, C_{\omega}$, and $C_{l}$ :

$$
\begin{aligned}
\mathbf{f}_{D} & =\frac{1}{2} C_{D} \pi r_{p}^{2} \rho\left|\mathbf{v}-\mathbf{v}_{p}\right|\left(\mathbf{v}-\mathbf{v}_{p}\right) ; \\
\mathbf{f}_{M} & =\frac{4}{3} C_{\omega} \pi r_{p}^{3} \rho\left[\left(\boldsymbol{\omega}-\boldsymbol{\omega}_{p}\right) \times\left(\mathbf{v}-\mathbf{v}_{p}\right)\right] ; \\
\mathbf{I}_{p} & =\frac{1}{2} C_{l} r_{p}^{5} \rho\left|\boldsymbol{\omega}-\boldsymbol{\omega}_{p}\right|\left(\boldsymbol{\omega}-\boldsymbol{\omega}_{p}\right)
\end{aligned}
$$

where $\boldsymbol{\omega}=(1 / 2)$ curl $\mathbf{v}$. The coefficients were calculated from the formulae approximating the analytical, experimental, and numerical data in wide ranges of the governing parameters of the flow around a single particle.

The drag coefficient $C_{D}$ was calculated from the approximation formula proposed in [10] for subsonic flow over a particle:

$$
\begin{aligned}
& C_{D}\left(\operatorname{Re}_{p}, \mathrm{M}_{p}, T_{p} / T\right) \\
& =24\left\{\operatorname{Re}_{p}+\sqrt{\frac{\gamma}{2}} \mathrm{M}_{p}\left[4.33+\frac{3.65-1.53 T_{p} / T}{1+0.353 T_{p} / T} \exp \left(-0.247 \sqrt{\frac{2}{\gamma}} \frac{\operatorname{Re}_{p}}{\mathrm{M}_{p}}\right)\right]\right\}^{-1} \\
& +\left[\frac{4.5+0.38\left(0.03 \mathrm{Re}_{p}+0.48 \sqrt{\mathrm{Re}_{p}}\right)}{1+0.03 \mathrm{Re}_{p}+0.48 \sqrt{\mathrm{Re}_{p}}}+0.1 \mathrm{M}_{p}^{2}+0.2 \mathrm{M}_{p}^{8}\right] \exp \left(-\frac{\mathrm{M}_{p}}{2 \sqrt{\mathrm{Re}_{p}}}\right) \\
& +0.6 \sqrt{\frac{\gamma}{2}} \mathrm{M}_{p}\left[1-\exp \left(-\frac{\mathrm{M}_{p}}{\operatorname{Re}_{p}}\right)\right] .
\end{aligned}
$$

Here, $\mathrm{M}_{p}=\left|\mathbf{v}-\mathbf{v}_{p}\right| / \sqrt{\gamma R T}$ and $\operatorname{Re}_{p}=2 \rho\left|\mathbf{v}-\mathbf{v}_{p}\right| r_{p} / \mu$ are the particle Mach and Reynolds numbers.

In the flow under consideration, the dependence of $C_{D}$ on $T_{p} / T$ is very weak; so, it was ignored and the ratio $T_{p} / T$ was taken equal to unity.

For calculation of $C_{\omega}$, the exact solution from [11] and the formula proposed in [12] were used:

$$
C_{\omega}= \begin{cases}\frac{3}{4}, & 2 \gamma_{\omega}<0.45 \\ \frac{3}{8} \hat{C}_{\omega}, & 2 \gamma_{\omega} \geq 0.45\end{cases}
$$

where

$$
\gamma_{\omega}=\frac{\left|\boldsymbol{\omega}-\boldsymbol{\omega}_{p}\right| r_{p}}{\left|\mathbf{v}-\mathbf{v}_{p}\right|}
$$




$$
\hat{C}_{\omega}\left(\gamma_{\omega}, \operatorname{Re}_{p}\right)=\frac{0.45+\left(2 \gamma_{\omega}-0.45\right) \exp \left(-0.075 \gamma_{\omega}^{0.4} \operatorname{Re}_{p}^{0.7}\right)}{\gamma_{\omega}}
$$

The expression for the coefficient $C_{l}$ was taken in the form proposed in [13]:

$$
C_{l}=\frac{C_{l 1}}{\sqrt{\operatorname{Re}_{p \omega}}}+\frac{C_{l 2}}{\operatorname{Re}_{p \omega}}
$$

where $\operatorname{Re}_{p \omega}=\rho\left|\boldsymbol{\omega}-\boldsymbol{\omega}_{p}\right| r_{p}^{2} / \mu$, and constants $C_{l 1}$ and $C_{l 2}$ are given in Table 1.
Table 1 Coefficients $C_{l 1}$ and $C_{l 2}$ in different ranges of the particle rotational Reynolds number $\operatorname{Re}_{p \omega}$

\begin{tabular}{ccc}
\hline $\mathrm{Re}_{p \omega}$ & $C_{l 1}$ & $C_{l 2}$ \\
\hline $0-6$ & 0 & $16 \pi$ \\
$6-20$ & 5.32 & 37.2 \\
$20-50$ & 6.44 & 32.2 \\
$50-40000$ & 6.45 & 32.1 \\
\hline
\end{tabular}

\subsection{Modeling of Particle-Blade Collisions}

Two particle-blade collision models, deterministic and stochastic, were considered in the present study. The first model was applied for spherical particles, and the second one for particles having the shape of a cube with cut vertices. In the last case, particles were assumed to have a random orientation relative to the blade surface before a collision. The collision was taken to be nonsliding and only partly elastic in both cases. In contrast to the case of a spherical particle, collision of a nonspherical particle with a blade is 3D. The present authors used the impact model described in detail in [14]. It is assumed that the impact interaction of a particle with a blade is instantaneous, and the particle position in space during the impact is fixed.

The equations of momentum and angular momentum for a particle have the form:

$$
m_{p}\left(\mathbf{v}_{p}^{+}-\mathbf{v}_{p}^{-}\right) \equiv m_{p} \Delta \mathbf{v}_{p}=\mathbf{S} ; \quad\left\|I_{p}\right\|\left(\boldsymbol{\omega}_{p}^{+}-\boldsymbol{\omega}_{p}^{-}\right) \equiv\left\|I_{p}\right\| \triangle \boldsymbol{\omega}_{p}=\mathbf{r}_{c} \times \mathbf{S} .
$$

Here, $\mathbf{S}$ is the impact momentum acting on the particle at the contact point; $\left\|I_{p}\right\|$ is the tensor of inertia of the particle; $\mathbf{r}_{c}$ is the position vector directed from the particle center of mass to the contact point (note that this vector is uniquely determined by particle orientation at the impact instant); and, as above, superscripts "-" and "+" denote the particle parameters before and after the impact.

Introduce the velocity $\mathbf{v}_{c}$ of the particle contact point. This vector is determined by the kinematic relation

$$
\mathbf{v}_{c}=\mathbf{v}_{p}+\omega_{p} \times \mathbf{r}_{c}
$$

From this, one can obtain:

$$
\triangle \mathbf{v}_{p} \equiv \mathbf{v}_{c}^{+}-\mathbf{v}_{c}^{-}=\mathbf{v}_{p}^{+}-\mathbf{v}_{p}^{-}+\triangle \boldsymbol{\omega}_{p} \times \mathbf{r}_{c} .
$$


Combining Eqs. (11) and (12) yields

$$
\frac{1}{m_{p}}\left\|I_{p}\right\| \triangle \boldsymbol{\omega}_{p}=\mathbf{r}_{c} \times \triangle \mathbf{v}_{c}-\mathbf{r}_{c} \times\left[\triangle \boldsymbol{\omega}_{p} \times \mathbf{r}_{c}\right] .
$$

Equation (13) contains two unknown vectors $\triangle \mathbf{v}_{c}$ and $\Delta \boldsymbol{\omega}_{p}$. Vector $\Delta \mathbf{v}_{p}$ can be determined if the restitution coefficients of the normal $\left(a_{n c}\right)$ and tangential $\left(a_{\tau c}\right)$ to the surface components of $\mathbf{v}_{c}$ defined by

$$
a_{n c}=-\frac{v_{c n}^{+}}{v_{c n}^{-}} ; \quad a_{\tau c}=\frac{v_{c \tau}^{+}}{v_{c \tau}^{-}}
$$

are known. Actually, these coefficients depend on the particle and surface materials, particle shape, collision angle, and precollisional particle parameters. For the model of nonsliding collision, the coefficient $a_{\tau c}$ is equal to zero. The coefficient $a_{n c}$ is taken to be constant. In this case, Eq. (13) is the equation for a single unknown $\Delta \boldsymbol{\omega}_{p}$. The method of solving this equation is described in [14]. The final relation for the velocity of the particle mass center after the collision is

$$
\mathbf{v}_{p}^{+}=\mathbf{v}_{p}^{-}+\triangle \mathbf{v}_{c}-\triangle \boldsymbol{\omega}_{p} \times \mathbf{r}_{c} .
$$

The equation for the postcollisional particle angular velocity is very cumbersome. The method of its solution is described in [14].

At the blade surface (at $\mathbf{r}=\mathbf{r}_{w}$ ), the boundary condition for the distribution function $f\left(\mathbf{r}, \mathbf{y}_{1}, t\right)$ is determined by a particle-blade collision model. In general case, valid for deterministic and stochastic collision models, the distribution function must satisfy the relation [5]

$$
\left|\mathbf{v}_{1} \cdot \mathbf{n}_{w}\right| f\left(\mathbf{r}, \mathbf{y}_{1}, t\right)=\int_{\mathbf{v}_{1}^{-} \cdot \mathbf{n}_{w}<0}\left|\mathbf{v}_{1}^{-} \cdot \mathbf{n}_{w}\right| W_{w}\left(\mathbf{y}_{1}^{-} \rightarrow \mathbf{y}_{1} \mid \mathbf{n}_{w}\right) f\left(\mathbf{r}_{w}, \mathbf{y}_{1}^{-}, t\right) d \mathbf{y}_{1}^{-}
$$

where $\mathbf{v}_{1} \cdot \mathbf{n}_{w}>o ; W_{w}\left(\mathbf{y}_{1}^{-} \rightarrow \mathbf{y}_{1} \mid \mathbf{n}_{w}\right)$ is the conditional distribution density of particle parameters $\mathbf{y}_{1}$ after rebound from the surface at the given values of parameters before the collision $\mathbf{y}_{1}^{-} ; \mathbf{n}_{w}$ is the unit vector normal to the surface and directed from the blade to the fluid. The density $W_{w}\left(\mathbf{y}_{1}^{-} \rightarrow \mathbf{y}_{1} \mid \mathbf{n}_{w}\right)$ is to be calculated on the basis of the collision model. If the particle parameters are being changed deterministically during a collision, according to the law $\mathbf{y}_{1}=\mathbf{y}_{w}^{+}\left(\mathbf{y}_{1}^{-}, \mathbf{n}_{w}\right)$, then $W_{w}\left(\mathbf{y}_{1}^{-} \rightarrow \mathbf{y}_{1} \mid \mathbf{n}_{w}\right)=\delta_{6}\left(\mathbf{y}_{1}-\mathbf{y}_{w}^{+}\left(\mathbf{y}_{1}, \mathbf{n}_{w}\right)\right)$ where $\delta_{6}$ is the 6-dimensional Dirac function. In the case of a stochastic collision model, the density $W_{w}\left(\mathbf{y}_{1}^{-} \rightarrow \mathbf{y}_{1} \mid \mathbf{n}_{w}\right)$ may be determined by the direct statistical simulation method based on calculations of a large number of collisions with random particle orientation prior to a collision. A similar technique was used in [14] for determination of scattering indicatrices of nonspherical particles rebounding from a smooth and a rough surface. 


\section{NUMERICAL METHOD}

As it was mentioned above, the two-phase gas-particle flow is considered without taking into account the action of the particle phase on the carrier gas flow. Two quite different approaches were used for simulating of the gas flow and the particle phase flow.

\subsection{Computational Fluid Dynamics Simulation of the Carrier Gas Flow}

Computational domain in (xy)-plane consisted of two blocks $A$ and $B$ (Fig. 2). Block $A$ moves together with the rotor blade and block $B$ is stationary. A curvilinear grid with quadrangular cells was constructed in the narrow layer fitted to the blade contour so that a number of cells across the boundary layer in the flow was about 20. An unstructured grid with triangular cells was generated outside this layer. A total number of grid cells in the moving block was about $10^{5}$ and in the stationary block about $1.5 \cdot 10^{5}$. Both grids were refined near the blade contours and behind them to provide a high resolution of flow structure in boundary layers and in the wakes. The total length of both blocks in the direction of an undisturbed flow was equal to $4.4 l$ ( $l$ is the airfoil

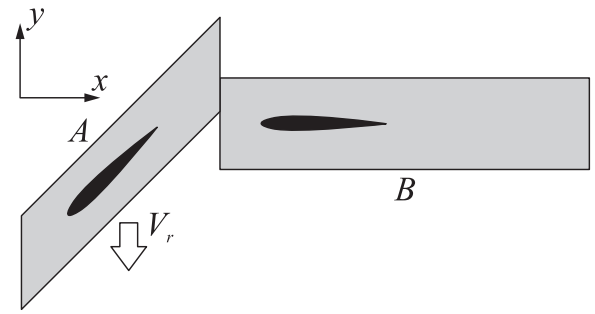

Figure 2 Computational domain chord).

A finite-volume method was used for solving the unsteady Navier-Stokes equations and the averaged Navier-Stokes equations with the Menter $k-\omega$ SST turbulence model. The details of this technique are described in [15].

At the inflow boundary of the moving block, two parameters, the total enthalpy and the entropy function

$$
h_{0}=\frac{\gamma}{\gamma-1} \frac{p_{\infty}}{\rho_{\infty}}+\frac{V_{\infty}^{2}}{2} ; \quad s=\frac{p}{\rho^{\gamma}},
$$

were specified. The static pressure $p$ was extrapolated from the computational domain. The velocity vector was assumed to be normal to the boundary. Then, the density and the velocity components can be calculated from the relations:

$$
\rho=\left(\frac{p}{s}\right)^{1 / \gamma} ; \quad u=\sqrt{2\left(h_{0}-\frac{\gamma}{\gamma-1} \frac{p}{\rho}\right)} ; \quad v=0 .
$$


In the case of URANS approach with the use of Menter $k-\omega$ SST turbulence model, the turbulent kinetic energy $k$ and $\omega$ were specified in addition:

$$
k=\frac{3}{2}\left(V_{\infty} I_{t}\right)^{2} ; \quad \omega=\frac{\rho k}{\mu_{t}} .
$$

In calculations, the turbulence intensity was taken $I_{t}=5 \%$ and the turbulent viscosity $\mu_{t}=(1 \ldots 10) \mu$.

At the outflow boundary of the stationary block, the static pressure was specified as $p_{\text {out }}=1.2 p_{\infty}$ and all other parameters were extrapolated from the computational domain.

Periodical in $y$-direction boundary conditions at the top and bottom boundaries of each block were applied. At the boundary between the blocks, a special matching procedure was used [15].

\subsection{Monte Carlo Simulation of the Particle Phase Motion}

Although the mathematical formulation of the problem for the particle phase includes the generalized Boltzmann equation (5) for the distribution function $f_{1}$ $=f\left(\mathbf{r}_{1}, \mathbf{v}_{p 1}, \boldsymbol{\omega}_{p 1}, t\right)$ with the collisional integral (6), the macroparameters $\langle\Phi\rangle(\mathbf{r}, t)$ of the dispersed phase, which are of principal interest, can be determined by the DSMC method without solving the kinetic equation (5). In this case, simulation of the particle phase behavior includes two steps: $(i)$ calculation of collisions between particles; and (ii) calculation of particle movement. There are several versions of this method. In the present study, the version described in detail in [6] was used.

Collisions and motion of particles were considered in 3D formulation within a 3D layer $1 \mathrm{~mm}$ thick in $z$-direction normal to the $(x, y)$-plane. At the side (parallel to $(x, y)$-plane) boundaries of this layer, the periodic in $z$-direction conditions were used: if any particle left the layer through a side boundary, it was excluded from consideration and a new particle with the same parameters was introduced into calculations at the opposite side. The number density of simulated particles in the undisturbed particle cloud was taken equal to that of real dispersed particles. Simulated particles were uniformly distributed in space in the undisturbed cloud.

Calculations of particles' motion were started up when the time-dependent flow of the carrier gas in the simulation reached a quasi-periodical regime. This delay is equal to the time required for the rotor cascade to travel a distance of about 30 pitches $s$ (see Fig. 1). The shape of particles in particle-blade collisions was taken either spherical or cubic with cut vertices; however, the particles of both shapes were considered as spherical in gas-particle interaction and in interparticle collisions. 


\section{COMPUTATIONAL RESULTS AND DISCUSSION}

The parameters of the cascades and flow properties in calculations were taken close to those which are typical for an inlet compressor of a turbojet engine. The chord of airfoils was equal to $l=10 \mathrm{~cm}$, cascade pitch $s=7 \mathrm{~cm}$, angle $\beta=45^{\circ}$, the profile of blades was NACA 0012, undisturbed flow velocity $V_{\infty}$ $=100 \mathrm{~m} / \mathrm{s}$, rotor cascade velocity $V_{r}=150 \mathrm{~m} / \mathrm{s}$, the carrier gas was air $(R$ $=287 \mathrm{~J} /(\mathrm{kg} \cdot \mathrm{K})$ with the ratio of specific heats $\left.c_{p} / c_{v}=1.4\right)$; Prandtl number $\operatorname{Pr}=0.72$, and flow density and temperature $\rho_{\infty}=1.21 \mathrm{~kg} / \mathrm{m}^{3}$ and $T_{\infty}=288 \mathrm{~K}$. These values correspond to Mach number $\mathrm{M}_{\infty}=0.3$. The particle material was silicon dioxide.

The carrier gas flow was visualized in two ways: by instant fields of the Mach number and the entropy function (Fig. 3) and also by computer animation of the

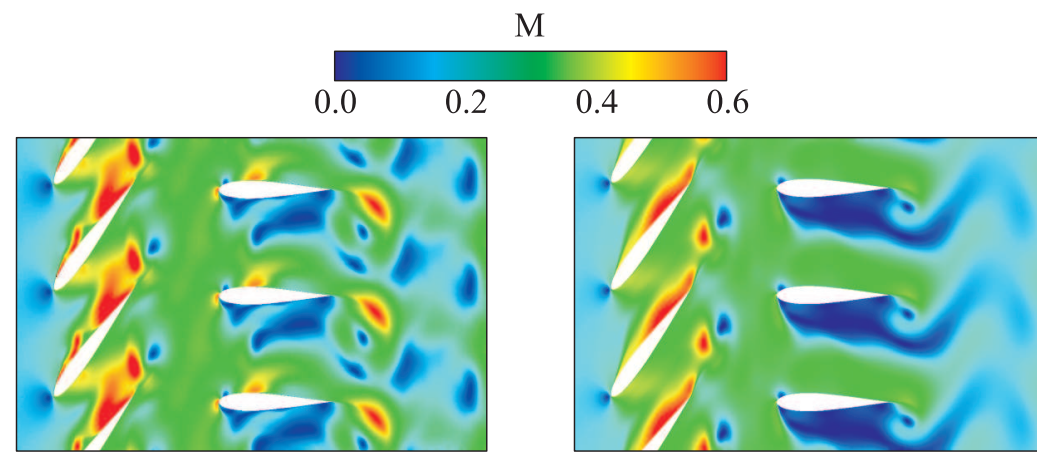

(a)

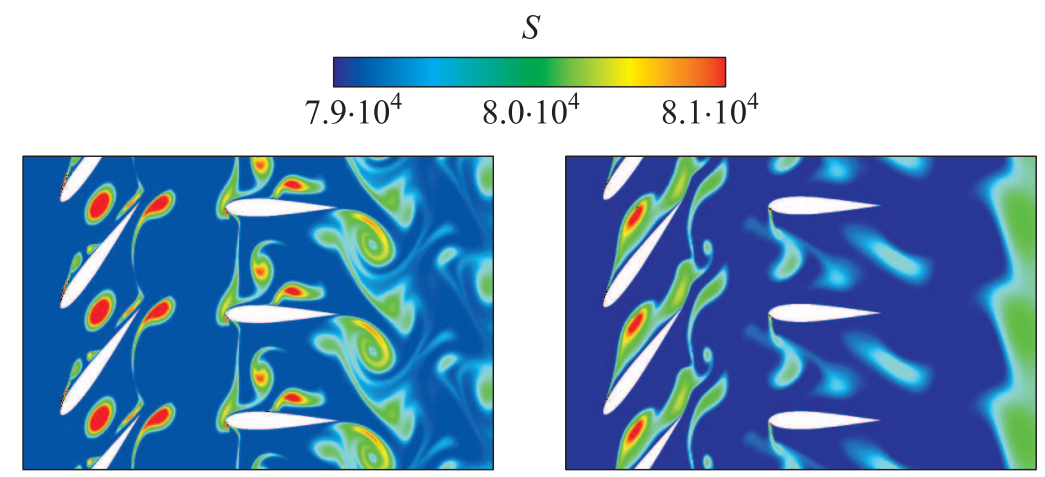

(b)

Figure 3 Instantaneous fields of Mach number $(a)$ and entropy function $S=p / p^{\gamma}(b)$ for the Navier-Stokes flow model (left column) and the $k-\omega$ SST turbulence model (right column) 
motion of massless marking particles. The latter way allowed to trace gas motion, in particular, separation and formation of eddies. As is seen from Fig. 3, the carrier gas flow structure is rather complex. The results obtained for the NavierStokes flow model and for the URANS flow model are qualitatively similar to each other. Large-scale eddies are formed and separated from blades. Note that simulation on the basis of the Navier-Stokes model gives a more detailed flow structure that is important for calculation of particles' motion because the actual flow parameters rather than the averaged ones determine the gas-particle interaction and, hence, the particle behavior. Although the calculations were performed with the use of the Navier-Stokes equations and the URANS approach with the Menter $k-\omega$ SST turbulence model, the results for particles are given below only for the Navier-Stokes flow model.

In the strict sense, a time-periodic solution for the carrier gas flow does not exist. It is connected with two independent processes periodic in time, namely, separation of eddies from blades and motion of spatially periodic rotor blades relative to the stator blades with the same pitch. The first process is determined by the Reynolds number of flow around a blade, whereas the second one is determined by the velocity $V_{r}$ and the cascade pitch $s$. If the periods of both processes are not multiple, their interaction can result in "stochastic" behavior of flow parameters with time. In computational simulation, a quasi-time-periodic flow was obtained.

The most impressive results are shown in Figs. 4-6. Patterns of a particle cloud consisting of spherical and cubic particles are shown for various instants of time in Figs. 4 and 5. Particles of sizes $20 \mu \mathrm{m}$ and $40 \mu \mathrm{m}$ correspond to Stokes numbers Stk $=3.41$ and 13.64, respectively. The Stokes number is defined as the ratio of the particle dynamic relaxation length (with the use of the Stokes law for a particle drag force) to the characteristic length in the flow $l$ : Stk $=\rho_{p} d_{p}^{2} V_{\infty} /\left(18 \mu_{\infty} l\right)$.

Spherical particles rebound from blades regularly, whereas cubic particles reflect stochastically. Scattering of cubic particles in their collisions with blades affects substantially the particle redistribution in the flow. The effect of collisions between particles which also results in their chaotic motion and redistribution in space, is much weaker even at rather high particle concentration. The value of particle volume fraction $\alpha_{p \infty}=3 \cdot 10^{-4}$ in Fig. 6 corresponds to the particle mass load on the order of unity in the undisturbed flow. Figure 6 shows the effect of interparticle collisions in forming the particle phase flow structure. As is seen from comparing Figs. $6 a$ and $6 b$ with the snapshots at the bottom of Figs. 4 and 5, respectively, the effect of collisions is remarkable for spherical particles which do not scatter in particle-blade collisions, whereas for cubic particles, this effect is negligible even at high particle concentration. This means that in the latter case, the redistribution of cubic particles due to their scattering in collisions with blades dominates substantially over the effect of interparticle collisions. 


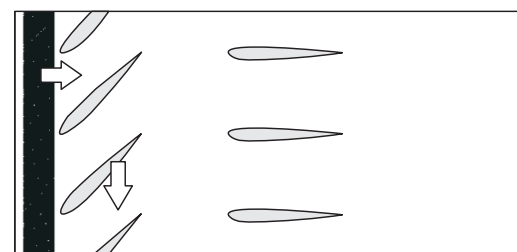

(a)

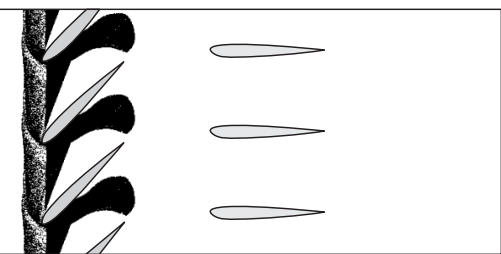

(b)

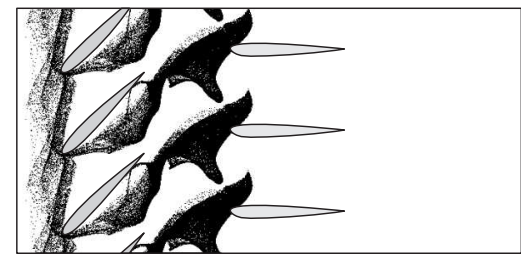

(c)

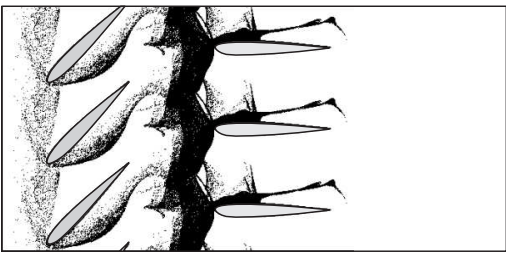

(d)


(e)



Figure 4 Instantaneous patterns of a particle cloud: spherical particles (left column) and cubic particles with cut vertices (right column). Particle size (diameter or edge) is $20 \mu \mathrm{m}$. The particle volume fraction is $10^{-4}$; interparticle collisions are not taken into account: $(a) t=0.5 \mathrm{~ms} ;(b) 1.5 ;(c) 2.5 ;(d) 3.5$; and $(e) t=5.0 \mathrm{~ms}$ 

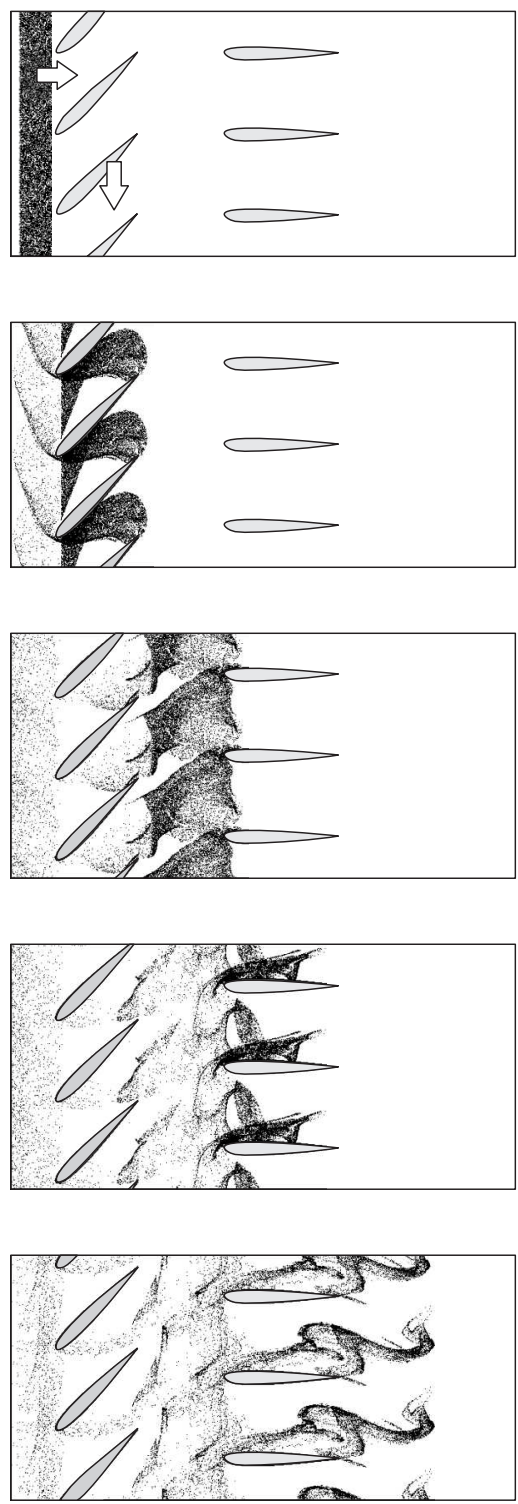

(e) (a)

(d)
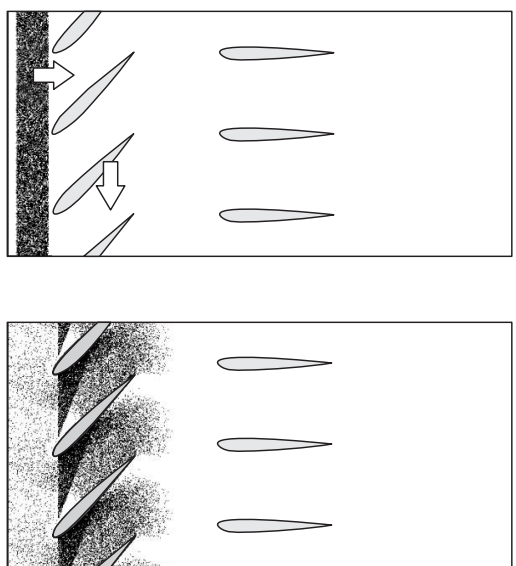

(b)

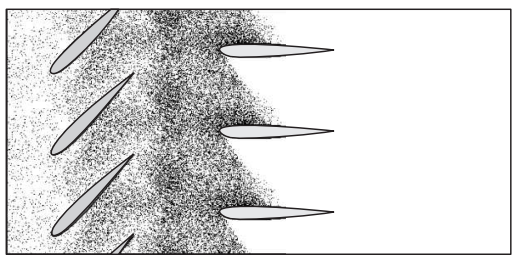

(c)
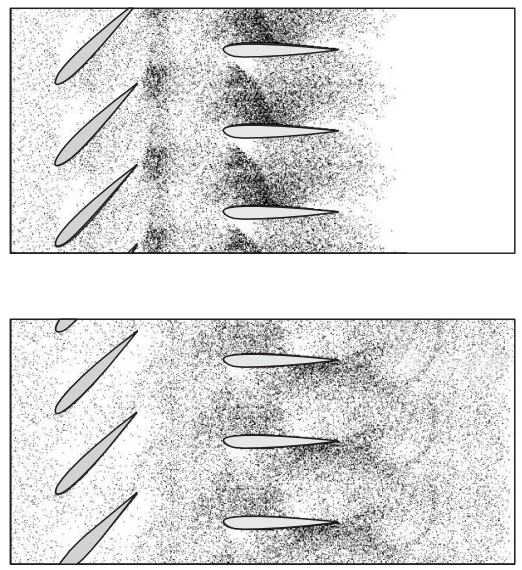

Figure 5 Instantaneous patterns of a particle cloud: spherical particles (left column) and cubic particles with cut vertices (right column). Particle size (diameter or edge) is $40 \mu \mathrm{m}$. The particle volume fraction is $10^{-4}$; interparticle collisions are not taken into account: $(a) t=0.5 \mathrm{~ms} ;(b) 1.5 ;(c) 2.5 ;(d) 3.5$; and $(e) t=5.0 \mathrm{~ms}$ 


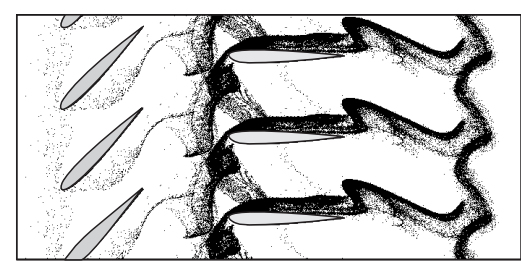

(a)
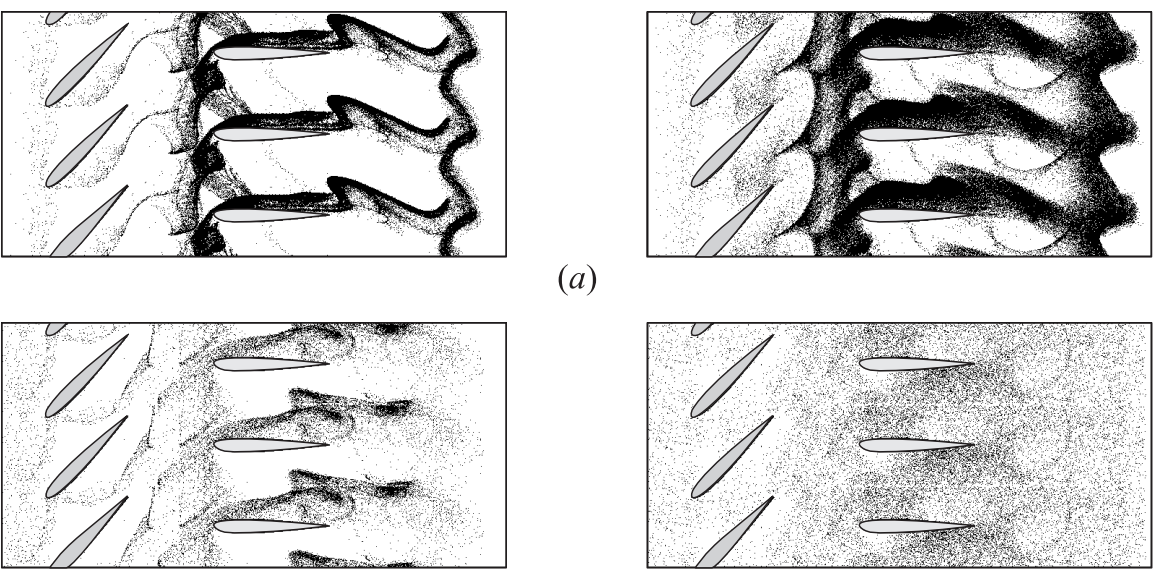

(b)

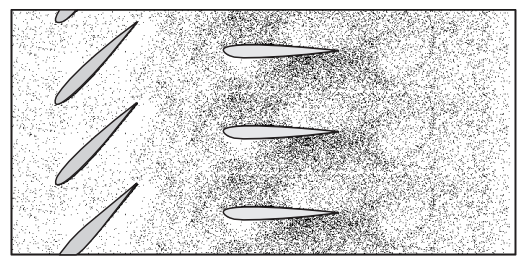

Figure 6 Instantaneous (at $t=5.0 \mathrm{~ms}$ ) patterns of a particle cloud: spherical particles (left column) and cubic particles with cut vertices (right column). The particle volume fraction is $3 \cdot 10^{-4}$; interparticle collisions are taken into account; particle size (diameter or edge) is $20(a)$ and $40 \mu \mathrm{m}(b)$

\section{CONCLUDING REMARKS}

The present investigation of dusty gas flow through a "rotor-stator" set of airfoil cascades has shown that dispersed particles are redistributed very strongly in the flow. The behavior of the particle phase depends on particle size and shape and on particle concentration. Spherical and nonspherical particles were considered. Spherical particles rebound regularly from blades and the main reason for them to move chaotically is the interparticle collisions. Nonspherical particles are scattered in particle-blade collisions and this effect for cubic particles with cut vertices turned out to be much stronger than the interparticle collisions. In spite of the fact that some "elementary" processes in the two-phase flow were considered in simplified formulation, the present results give a good estimation of the role of such important effects as the collision between particles and the scattering of nonspherical particles in their impact interaction with the blades.

\section{ACKNOWLEDGMENTS}

The authors wish to express their sincere gratitude to Dr. S. V. Panfilov for the help in performance of computations and discussion of results. This work was supported financially by the Russian Foundation for Basic Research through grants Nos. 09-08-00888 and 12-08-01282. 


\section{REFERENCES}

1. Stepanov, G. Yu. 1962. Hydrodynamics of cascades of turbomachines. Moscow: Fizmatlit. 512 p. (In Russian.)

2. Healy, D. P., and J. B. Young. 2005. Full Lagrangian methods for calculating particle concentration fields in dilute gas-particle flows. Proc. R. Soc. A 461:2197-2225.

3. Tsirkunov, Yu. M., D. A. Romanyuk, and S. V. Panfilov. 2011. Effects of particle mixing and scattering in the dusty gas flow through moving and stationary cascades of airfoils. Progress in propulsion physics. Eds. L. DeLuca, C. Bonnal, O. Haidn, and S. Frolov. TORUS PRESS / EDP Sciences. 2:459-474.

4. Tsirkunov, Yu. M. 2001. Gas-particle flows around bodies - key problems, modeling and numerical analysis. 4th Conference (International) on Multiphase Flow (ICMF'2001) Proceedings. Ed. E. Michaelides. Paper No.607. 31 p. CD-ROM.

5. Volkov, A. N., and Yu. M. Tsirkunov. 2000. Kinetic model of a collisional admixture in dusty gas and its application to calculating flow past bodies. Fluid Dyn. 35(3):380-392.

6. Volkov, A.N., and Yu.M. Tsirkunov. 2002. CFD/Monte Carlo simulation of collision-dominated gas-particle flows over bodies. ASME FEDSM'2002 Proceedings. Paper No. 31222. 14 p.

7. Menter, F. R. 1994. Two-equation eddy-viscosity turbulence models for engineering applications. AIAA J. 32(8):1598-1605.

8. Menter, F., J. C. Ferreira, T. Esch, and B. Konno. 2003. The SST turbulence model with improved wall treatment for heat transfer predictions in gas turbines. Gas Turnine Congress (International) Proceedings. Tokyo. Paper No. IGTC2003-TS059. $7 \mathrm{p}$.

9. Tannehill, J. C., D. A. Anderson, and R.H. Pletcher. 1997. Computational fluid mechanics and heat transfer. 2nd ed. Taylor \& Francis. 816 p.

10. Henderson, Ch. B. 1976. Drag coefficients of spheres in continuum and rarefied flows. AIAA J. 14(6):707-708.

11. Rubinow, S.I., and J.B. Keller. 1961. The transverse force on a spinning sphere moving in a viscous fluid. J. Fluid Mech. 11:447-459.

12. Oesterlé, B., and T. Bui Dinh. 1998. Experiments on the lift of a spinning sphere in a range of intermediate Reynolds numbers. Exp. Fluids 25:16-22.

13. Dennis, S. C.R., S. N. Singh, and D. B. Ingham. 1980. The steady flow due to a rotating sphere at low and moderate Reynolds numbers. J. Fluid Mech. 101:257279.

14. Panfilov, S. V., and Yu.M. Tsirkunov. 2008. Scattering of nonspherical particles rebounding from a smooth and a rough surface in a high-speed gas-particle flow. J. Appl. Mech. Tech. Phys. 49(2):222-230.

15. Romanyuk, D. A., and Yu. M. Tsirkunov. 2010. Numerical simulation if unsteady dusty gas flow through the moving and stationary cascades of airfoils. 5th European Conference on Computational Fluid Dynamics: ECCOMAS CFD 2010 Proceedings. Eds. J. C. F. Pereira and A. Sequeira. Paper No. 1063. 20 p. CD-ROM. 